\title{
Diacronie
}

Studi di Storia Contemporanea

$N^{\circ} 17,1 \mid 2014$

Periferie. Cultura, economia, politica

\section{Eric Gobetti, Alleati del nemico. L'occupazione italiana in Jugoslavia (1941-1943}

\section{Mario De Prospo}

\section{(2) OpenEdition}

Edizione digitale

URL: http://journals.openedition.org/diacronie/1140

DOI: 10.4000/diacronie. 1140

ISSN: 2038-0925

Editore

Association culturelle Diacronie

Notizia bibliografica digitale

Mario De Prospo, "Eric Gobetti, Alleati del nemico. L'occupazione italiana in Jugoslavia (1941-1943 », Diacronie [Online], $N^{\circ}$ 17, 1 | 2014, documento 13, online dal 01 mars 2014, consultato il 21 décembre 2020. URL : http://journals.openedition.org/diacronie/1140; DOI : https://doi.org/10.4000/diacronie. 1140 


\section{Diacronie}

N. 17 | $1 \mid 2014$ Periferie. Cultura, economia, politica

13/

\section{RECENSIONE:}

\section{Eric GOBETTI, Alleati del nemico. L'occupazione italiana in Jugoslavia (1941- 1943), Roma-Bari, Laterza, 2013, 205 pp.}

a cura di Mario DE PROSPO *

Questo volume è il punto di arrivo di oltre dieci anni di ricerche, attraverso le quali Eric Gobetti, partendo dal periodo di occupazione italiana dei territori dell' exJugoslavia e dalle vicende belliche che la sconvolsero, ha provato a far comprendere la storia di questa complessa area del vecchio continente ${ }^{1}$.

Si ritiene opportuno sottolineare che questo contributo esce circa un anno dopo la pubblicazione del lavoro di Elena Aga Rossi e Maria Teresa Giusti che si è già avuto modo di presentare ${ }^{2}$. Entrambe le opere, infatti, trattano della presenza dei militari italiani nei territori balcanici, ma sono differenti i focus principali. Mentre Aga Rossi e Giusti si concentrano soprattutto sulla crisi che affrontarono i reparti del regio esercito al momento dell'annuncio dell'armistizio; Gobetti, invece, dedica il suo lavoro a quanto succede prima dell'8 settembre 1943, concentrandosi sull'occupazione di questi territori da parte delle truppe del nostro paese.

Le caratteristiche che contraddistinguono lo scacchiere jugoslavo tra il 1941 e il 1943 sono sicuramente la complessità e l'ambiguità. È lo stesso autore a premettere che

${ }^{1}$ Cfr. GOBETTI, Eric, Dittatore per caso. Un piccolo duce protetto dall'Italia fascista, Napoli, L'Ancora del Mediterraneo, 2001; ID., L'occupazione allegra. Gli italiani in Jugoslavia (19411943), Roma, Carocci, 2007; si ritiene utile menzionare anche questo testo dell'autore, testimonianza dell'interesse a $360^{\circ}$ per i territori della ex-Jugoslavia: ID. Nema problema! Jugoslavie! 10 anni di viaggi, Torino, Miraggi, 2011.

2 Cfr. AGA ROSSI, Elena, GIUSTI, Maria Teresa, Una guerra a parte. I militari italiani nei Balcani 1940-1945, Bologna, Il Mulino, 2011, Cfr. DE PROSPO, Mario, «Recensione: Elena AGA ROSSI, Maria Teresa GIUSTI, Una guerra a parte. I militari italiani nei Balcani 1940-1945, Bologna, Il Mulino, 2011, 66o pp.», in Diacronie. Studi di Storia Contemporanea: La satira fa storia. Eventi, pratiche, linguaggi, 29 ottobre 2012, URL:

< http://www.studistorici.com/2012/10/29/de-prospo_numero_11/ >. È giusto rilevare che il volume dell'Aga Rossi e della Giusti abbraccia un'area territoriale più vasta oltre quella jugoslava, comprendente anche Grecia e Albania. 
quanto succede nei Balcani del sud con il loro coinvolgimento nella Seconda guerra mondiale, non differentemente da molti altri pesi dell'Est europeo, «resta tutt'oggi l'evento cardine della storia dei popoli jugoslavi, ora perlopiù interpretato nella logica della guerra civile, sminuendo il valore morale della resistenza all'invasore»3. In questo ingarbugliato contesto si trovano le truppe italiane a partire dall'aprile del 1941. Una presenza, quella dei nostri connazionali, che non può essere considerata come quella di semplici spettatori di quanto succede in questa regione. Infatti, come sottolinea opportunamente l'autore, sebbene sia stata rimossa dalla memoria collettiva dei nostri connazionali4, l'idea dell'occupazione di questi territori parte da lontano, una precisa visione strategica, affinata negli anni precedenti, di un Mediterraneo egemonizzato dall'Italia - in cui lo Stato Jugoslavo nato dopo la Grande Guerra rappresenta un antagonista e un ostacolo per il predominio dell'Adriatico - che porta all'allontanamento dalla tradizionale politica filo-serba che aveva caratterizzato l'età liberale, in favore del sostegno alle aspirazioni albanesi e croate, che dovrebbero diventare i pilastri dell'influenza italiana della regione5. Aspirazioni che si traducono concretamente con l'occupazione dell'Albania nel 1939 e la costituzione dello stato croato guidato dal "protetto" Ante Paveli, nel momento stesso dell'invasione della Jugoslavia, al fianco dei tedeschi, nella primavera del 1941.

Le ambizioni italiane si devono misurare con una situazione che, mese dopo mese, diventa ancora più complicata. Il controllo del territorio si rivela difficile: il dialogo con le preesistenti élites locali è controproducente e risultati migliori non arrivano neppure dalla collaborazione con la neonata Croazia guidata da Paveli . Il crollo dell'entità statale jugoslava fa esplodere aspri conflitti lungo fratture sia etniche, che politiche.

Nel giro di pochi mesi gli italiani sono costretti a cambiare strategia: gli interessi economici e politici passano in secondo piano e la nostra presenza-diventa soprattutto militare, tanto che il Regio Esercito, ad inizio 1942, arriva ad avere oltre 300.000 soldati nella regione. Questa evoluzione viene orientata e condivisa dallo stesso Mussolini, per cui l'occupazione si tramuta in «una politica imperialista di stampo ottocentesco, basata sul controllo militare del territorio e delle risorse piuttosto che sull'egemonia politico-economica ${ }^{6}$.

\footnotetext{
3 GOBETTI, Eric, Una guerra a parte. I militari italiani nei Balcani 1940-1945, Roma-Bari, Laterza, 2013,p. 9.

4 Ibidem, p. 7.

5 Ibidem, pp.14-15 . Cfr. anche RODOGNO, Davide, Il nuovo ordine mediterraneo. Le politiche di occupazione dell'Italia fascista (1940-43), Torino, Bollati Boringhieri, 2003.

${ }^{6}$ GOBETTI, Eric, Alleati del nemico, cit., p. 76.
} 
In questo contesto i principali avversari si rivelano le forze partigiane comuniste comandate da Tito, in ascesa dopo l'invasione dell'URSS e il coinvolgimento diretto nella guerra della potenza guidata da Stalin, con cui si deve ingaggiare una logorante azione repressiva. Contemporaneamente, per poter controllare e amministrare i territori occupati, i vertici in grigioverde promuovono l'alleanza con un ampio fronte locale anticomunista, che porta nel 1942 all'istituzione della Milizia Volontaria Anticomunista (Mvac), che formalizza i rapporti di cooperazione con le milizie cetniche, avviati ormai da diversi mesi. Da qui il titolo dell'opera, Alleati del nemico, perché le milizie cetniche, sono espressione del nazionalismo serbo di stampo conservatore, che immagina una Jugoslavia egemonizzata dai serbi. Il capo di queste milizie è Dra a Mihailovi , ministro della guerra del governo monarchico jugoslavo, in esilio a Londra dopo l'invasione dell'Asse. I cetnici, infatti, oltre a contrapporsi ai comunisti, combattono duramente, sin all'inizio dell'occupazione nazifascista, contro le forze croate dell'alleato Ante Paveli .

All'interno di questa complicata e contraddittoria rete di alleanze, i vertici italiani chiedono alle proprie truppe vigore e spregiudicatezza, per smentire lo stereotipo, già allora percepito, del buon soldato italiano ${ }^{7}$. In questo contesto si spiega la circolare ${ }_{3} \mathrm{C}$ emanata dal generale Mario Roatta nel marzo del 1942, che rimarrà in vigore fino all'armistizio. Con questa circolare gli italiani accolgono «esplicitamente il principio di correità della popolazione residente in un'area di attività partigiana» e fanno proprio «come metodo la politica del terrore contro i civili, ordinando rappresaglie, deportazioni, confische, catture di ostaggi, fucilazioni» ${ }^{8}$. Strumenti principali della strategia repressiva, ricorda Gobetti, sono l'internamento dei civili, che arrivano alla cifra 100.00o individui deportati, in gran parte sloveni e montenegrini ${ }^{9}$ e l'utilizzo della tattica della terra bruciata, ovvero la distruzione di case e villaggi per rappresaglia. I metodi del Regio Esercito, sottolinea l'autore, ricordano da vicino quelli attuati negli

\footnotetext{
7 Cfr. GOBETTI, Eric, L'occupazione allegra, cit.,pp.173-176. Sulla creazione e del 'mito' del buon italiano la bibliografia è corposa, segnaliamo su questi argomenti il recente FOCARDI, Filippo, Il cattivo tedesco e il bravo italiano, la rimozione delle colpe della seconda guerra mondiale, Roma-Bari, Laterza, 2013. Cfr. la recensione del volume in VERONESI, Oreste, «Recensione: Filippo FOCARDI, Il cattivo tedesco e il bravo italiano. La rimozione delle colpe della seconda guerra mondiale, Roma-Bari, Laterza, 2013, XIX + 288 pp.», Diacronie. Studi di Storia Contemporanea : Spazi, percorsi e memorie, 29/10/2013. URL:< http://www.studistorici.com/2013/10/29/veronesi_numero_15/>.

8 GOBETTI, Eric, Alleati del nemico, cit., p. 91.

9 Ibidem, p. 95. Cfr. CAPOGRECO, Carlo Spartaco, I campi del duce. L'internamento nell'Italia Fascista (190-43), Torino, Einaudi, 2004, pp.135-152; KERSEVAN, Alessandra, Lager italiani. Pulizia etnica e campi di concentra mento fascisti per civili jugoslavi 1941-1943, Roma, Nutrimenti, 2008.
} 
scenari coloniali ${ }^{10}$. In questo contesto e in base a queste scelte, vengono compiuti crimini efferati anche dai militari italiani, senza che l'intera strategia attuata vada incontro a critiche. Al contrario, chi nel dopoguerra è stato inquisito per i crimini commessi in Jugoslavia si è autodifeso sostenendo che l'escalation di violenza sia stata causata dalla brutalità dei partigiani comunisti, una convinzione - quella della violenza dei comunisti jugoslavi - entrata nell'immaginario del nostro paese, che però, ci tiene a sottolineare l'autore, non trova riscontro nelle fonti relative a questo periodo, con le dovute tragiche eccezioni ${ }^{11}$. La memoria dei reduci è sicuramente influenzata da questa situazione, che farà il paio con la sopraggiunta difficoltà nel collocare questa esperienza in un dopoguerra che vedrà l'esaltazione, nei partiti di sinistra, della retorica partigiana. Per questo motivo nei volumi di ricordi di quest'esperienza, secondo Gobetti, emergono, da parte italiana, due elementi, a suo avviso solo apparentemente contraddittorii: «una quotidianità annoiata di cui si evidenziano soprattutto i buoni rapporti stabiliti con la popolazione civile; una serie di improvvisi eventi drammatici, che mostrano la brutalità della guerriglia ${ }^{12}$. L'autore sostiene che questa memoria contraddittoria viene alimentata anche dal crescente spaesamento dei soldati italiani, che vedono crescere i consensi a favore della resistenza comunista, dimostratasi spesso molto più umana delle altre parti in conflitto, spuntando così le armi della propaganda anti-partigiana. Allo stesso tempo, i nostri soldati fanno fatica a comprendere la presenza di diverse e spesso contrapposte milizie locali, che si rendono frequentemente protagoniste di spietati atti di violenza, a sfondo etnico, compiuti proprio davanti ai loro occhi senza la possibilità di intervenire ${ }^{13}$, con l'unica eccezione di diverse migliaia di ebrei messi-in salvo con il contributo del Regio Esercito ${ }^{14}$.

Con il passare dei mesi diventa sempre più difficile, davanti ai croati e ai tedeschi, giustificare da parte dei vertici italiani, l'alleanza con quelli che sono, ufficialmente, i nemici cetnici. Una situazione che favorisce un crescente avvicinamento degli ustascia di Paveli ai tedeschi.

Il capovolgersi complessivo delle sorti della guerra, con le vittorie in Nord Africa degli anglo-americani e, nel giro di pochi mesi, il passaggio all'offensiva dei Sovietici rende consci i comandanti italiani che è necessario cominciare un disimpegno dall'area,

${ }^{10}$ GOBETTI, Eric, Alleati del nemico, cit., p. 100. Cfr. anche RODOGNO, Davide, op. cit., pp. 400-410; SALA, Teodoro, Guerra e amministrazione in Jugoslavia 1941-1943. Un'ipotesi coloniale in POGGIO, Pier Paolo, MICHELETTI, Bruna (a cura di), L'Italia in guerra 1940-43, Brescia, Fondazione Luigi Micheletti, 1992, pp. 83-97.

${ }^{11}$ GOBETTI, Eric, Alleati del nemico, cit., p. 104.

${ }^{12}$ Ibidem, p. 108

13 Ibidem, p. 115.

14 Ibidem, pp. 138-141. 
per prepararsi all'imminente difesa dei confini nazionali. Il colpo di grazia alla strategia dei generali del Regio Esercito nei territori Jugoslavi arriva tra marzo e giugno del 1943 con la sconfitta nella battaglia della Neretva, in Erzegovina. I partigiani di Tito riescono a sfuggire all'accerchiamento delle truppe nazi-fasciste e sbaragliano i cetnici, imponendosi definitivamente come i protagonisti della resistenza nell'area ${ }^{15}$. Le sorti della guerra, in particolar modo quelle dell'Italia con la contemporanea definitiva sconfitta in Tunisia, a questo punto sono irrimediabilmente segnate. "All'annuncio dell'Armistizio, i soldati italiani in Jugoslavia possono contare solo su stessi» e gran parte di essi verranno fatti prigionieri dai tedeschi ${ }^{16}$.

L'autore basa tutta la ricerca su un impressionante apparato di fonti primarie e secondarie, di cui la documentazione italiana è solo una, seppur importante, parte. Il risultato, oltre ad un ulteriore ed utile approfondimento sulla presenza italiana e le politiche di occupazione attuate dai nostri connazionali, è un'efficace interpretazione dei motivi per cui il conflitto ha avuto determinati esiti in questa parte d'Europa, con valutazioni che vanno ben oltre il piano dei rapporti di forza militari.

Il passo ulteriore che l'autore sviluppa in questo testo, rispetto alle sue opere già pubblicate su questo tema, è il tentativo di inserire con precisione le ricerche nel più ampio contesto della Seconda guerra mondiale e, contemporaneamente, fornire una lettura agevole e di sintesi su vicende delicate e particolarmente ricche di sfaccettature ed elementi contraddittori. 


\section{* L'autore}

Mario De Prospo si è addottorato in Storia Contemporanea presso L'Istituto Italiano di Scienze Umane - SUM (Firenze, Napoli), dove ha svolto una ricerca dal titolo Mezzogiorno 1943. Soldati allo sbando sotto la tutela di Paolo Macry. Ha conseguito la laurea magistrale nel 2007 presso l'Università degli Studi di Napoli - Federico II con una tesi in Storia Contemporanea dal titolo Dall'asse alla cobelligeranza. I prigionieri di guerra italiani negli Stati Uniti (rel. Paolo Macry).

URL: < http://www.studistorici.com/progett/autori/\#DeProspo >

\section{Per citare questo articolo:}

DE PROSPO, Mario, «Recensione: Eric GOBETTI, Alleati del nemico. L'occupazione italiana in Jugoslavia (19411943), Roma-Bari, Laterza, 2013, 205 pp.», Diacronie. Studi di Storia Contemporanea : Periferie. Cultura, economia, politica, 29/03/2014,

URL: < http://www.studistorici.com/2014/3/29/deprospo_numero_17/ >

\section{Diacronie Studi di Storia Contemporanea $\mathcal{B}$ www.diacronie.it}

Risorsa digitale indipendente a carattere storiografico. Uscita trimestrale. redazione.diacronie@hotmail.it

Comitato di redazione: Jacopo Bassi - Luca Bufarale - Elisa Grandi - Deborah Paci - Fausto Pietrancosta - Matteo Tomasoni - Luca Zuccolo 